Ann. Génét. Sél. anim., I973, 5 (4), 45I-46I.

\title{
LES PENDANTS D'OREILLES DANS LA RACE OVINE SARDE ( $\left.{ }^{1}\right)$
}

\author{
J. G. BOYAZOGLU, S. CASU * et J.- J. LAUVERGNE **
}

avec la collaboration technique de P. Carta, G. Picinelli, G. Ruda et L. Sanna

\author{
Animal and Dairy Science Research Institute, \\ Private Bag 177, \\ Pretoria, South Africa \\ * Istituto Zootecnico e Caseario per la Sardegna, \\ Casella Postale 07100. \\ Sassari, Italia \\ ** Laboratoire de génétique factorielle, \\ Centre national de Recherches zootechniques, I. N.R. A., \\ 78350 Jouy en Josas
}

\section{RÉSUMÉ}

Les pendants d'oreilles (" orechiete") ont, en race ovine Sarde, des formes, des localisations et des associations variables, mais on peut relier leur présence à l'action d'un seul facteur mendélien autosomal à pénétrance incomplète et expressivité variable. Le sexe n'a pas d'influence sur les pénétrances que l'on a pu estimer chez l'homozygote $\left(w_{1}=0,85\right)$ et chez l'hétérozygote $\left(w_{2}=0,43\right)$.

La fréquence génique, estimée à partir d'un échantillon de croisements entre animaux non porteurs des troupeaux de l'Institut Zootechnique et Fromager Sarde d'où proviennent les données qui ont permis d'établir le déterminisme héréditaire, est faible $: q=5 \cdot{ }^{\cdot}$ Io $^{-4}$. Cette estimée, par défaut, est proche de celle observée dans les troupeaux inscrits des deux provinces de Cagliari et de Sassari d'où proviennent d'ailleurs les animaux de l'Institut $: q=8,5^{\cdot} \mathrm{Io}^{-4}$. La fréquence dans l'autre province sarde de Nuoro diffère significativement de celle observée à Cagliari et Sassari. Cela peut provenir du fait que, comme on l'a montré dans une précédente étude les troupeaux de ces 2 provinces sont fortement influencés génétiquement par le centre de Barumini. Dans la province de Nuoro, par contre, le courant migrateur des béliers est plus faible. Dans les 3 provinces, par ailleurs, les fréquences du gène dans les troupeaux non inscrits sont significativement plus élevées que dans les troupeaux inscrits. Un choix beaucoup plus diversifié des béliers dans les troupeaux non inscrits peut expliquer cette hétérogénéité des fréquences géniques.

(1) Une première communication sur ce sujet a été faite au Congrès Européen de Zootechnie à Versailles, en juillet I97I (CASU et al., I97I). Elle a été distribuée sous forme de tiré à part lors des sessions mais ne sera pas reprise dans des comptes rendus reliés. Elle est, par ailleurs, beaucoup moins complète que le présent article. 

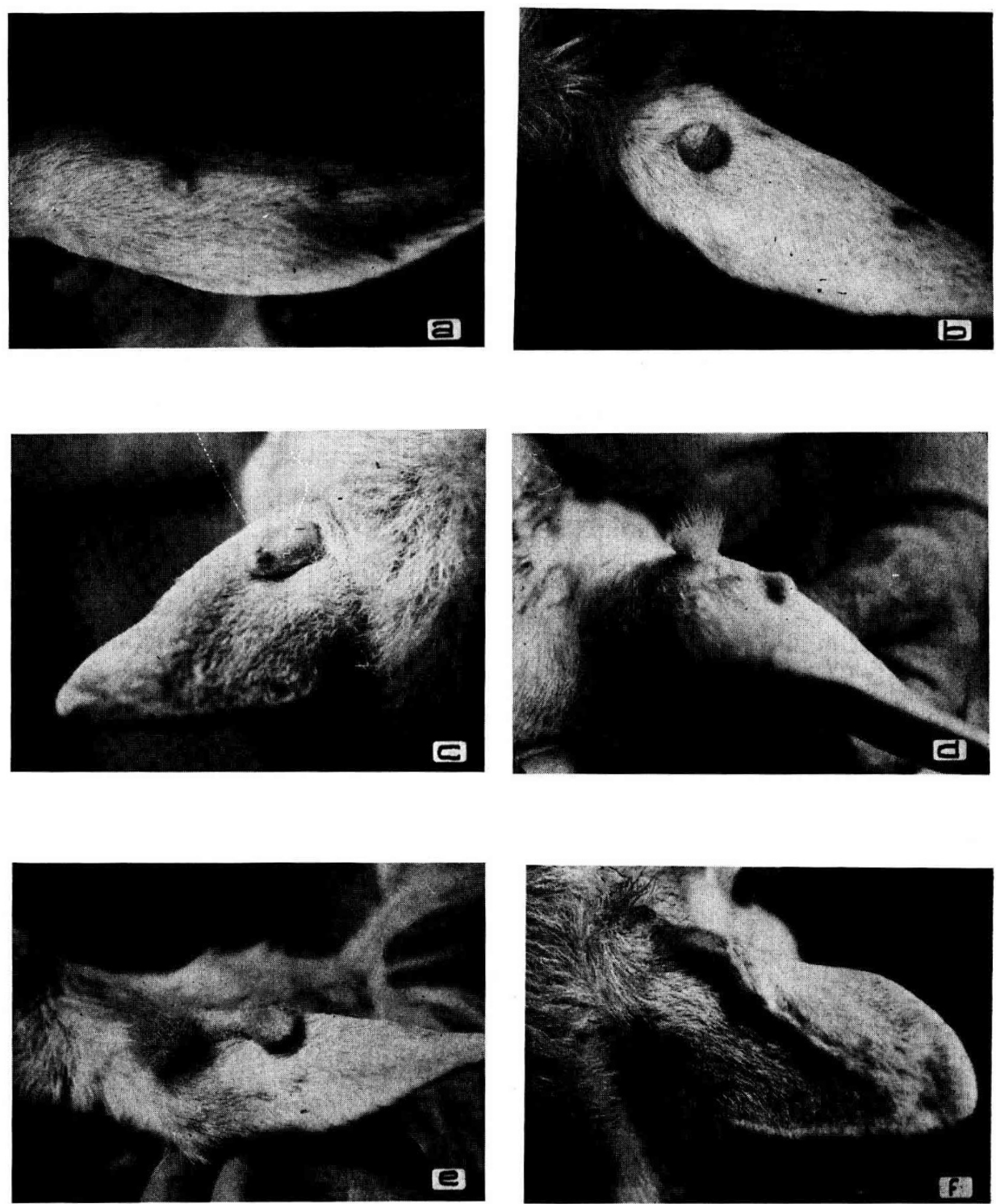

Fig. I. - Formes et localisation des pendants d'oreilles en race Sarde

a) Un seul pendant tout petit;

b) Un seul pendant pisiforme moyennement gros ;

c) Un seul pendant allongé;

d) Deux pendants de formes différentes;

e) Deux pendants reliés entre eux par un pont cartilagineux;

f) Formation allongée en " peigne" (piega). 


\section{INTRODUCTION}

Les pendants d'oreilles sont de petites formations cartilagineuses recouvertes de peau et localisées sur la face supérieure de l'oreille externe des moutons. Leur étiologie est donnée comme héréditaire dans cette espèce (VASIN, I928, SERRA, I948).

En Sardaigne on les rencontre parfois dans la race ovine autochtone où on les nomme " orechiette" (petites oreilles).

La présente recherche est consacrée à l'étude de leur déterminisme héréditaire et de leur fréquence dans cette population. C'est le troisième d'une série de travaux réalisés à l'Institut Zootechnique et Fromager Sarde (Istituto Zootecnico e Caseario per la Sardegna, siège à Sassari) et consacrés à la race ovine Sarde du point de vue de la génétique mendélienne et de la génétique des populations. Le premier concernait une étude sur les pendeloques (CASU et al., I970) et le second portait sur la structure génétique de la population insulaire (LAUVERGNE et al., I973).

\section{MATÉRIEL ANIMAL,}

\section{Le matériel pour l'étude du mode de transmission}

Il provient de croisements expérimentaux réalisés dans les fermes de l'Institut (Bonassai, Foresta di Burgos et Monastir) à partir d'animaux munis de ces attributs en provenance de troupeaux inscrits au Flock-book dans les provinces de Sassari et de Cagliari. Un bélier porteur et deux de ses fils porteurs ont ainsi été employés, ainsi que 29 brebis présentant le caractère. En trois campagnes de mise bas $(1970,197$ I, 1973) 49 produits issus de croisement porteur $\times$ porteur, et I 98 du type porteur par non porteur ont été examinés cependant que, dans ces mêmes troupeaux expérimentaux, on contrôlait ${ }_{4} 226$ naissances provenant d'accouplements entre animaux non porteurs.

\section{L'enquête démographique}

Au cours de l'enquête démographique générale de 1970 analysée dans l'article de LAUVERGNE et al. (1973) on a recueilli des données relatives à la fréquence du caractère dans les populations insulaires dans 137 troupeaux inscrits sur un total de I6I pour les trois provinces de l'île. Les comptages ont été faits également dans I35 troupeaux non inscrits localisés au voisinage des troupeaux inscrits inventoriés et qui ont servi de témoins.

\section{MÉTHODES D'ANALYSE E'T RÉSULTATS}

\section{I. - Les modes d'expression du caractère}

Les formations qui nous intéressent présent: nt une expressivité variable dont les principaux types sont présentés dans la figure I.

On note tout d'abord des formations simples (fig. I $a, b, c$ ) de taille variable, des formations doubles à pendants isolés (fig. I $d$ ) ou reliés par un pédoncule (fig. I $e$ ) le dernier stade étant une sorte de languette appelée " peigne " dans laquelle le pédoncule a pris une importance très grande (fig. I $f$ ).

\section{2. - Les formations étudiées sont transmises héréditairement}

Cela se déduit avec suffisamment d'évidence de l'examen du tableau I qui donne les résultats des croisements expérimentaux. 
TABLEAU I

Résultats des accouplements expérimentaux et des observations dans les troupeaux de l'Institut Zootechnique et Fromager Sarde pour l'étude du caractère pendants d'oreilles

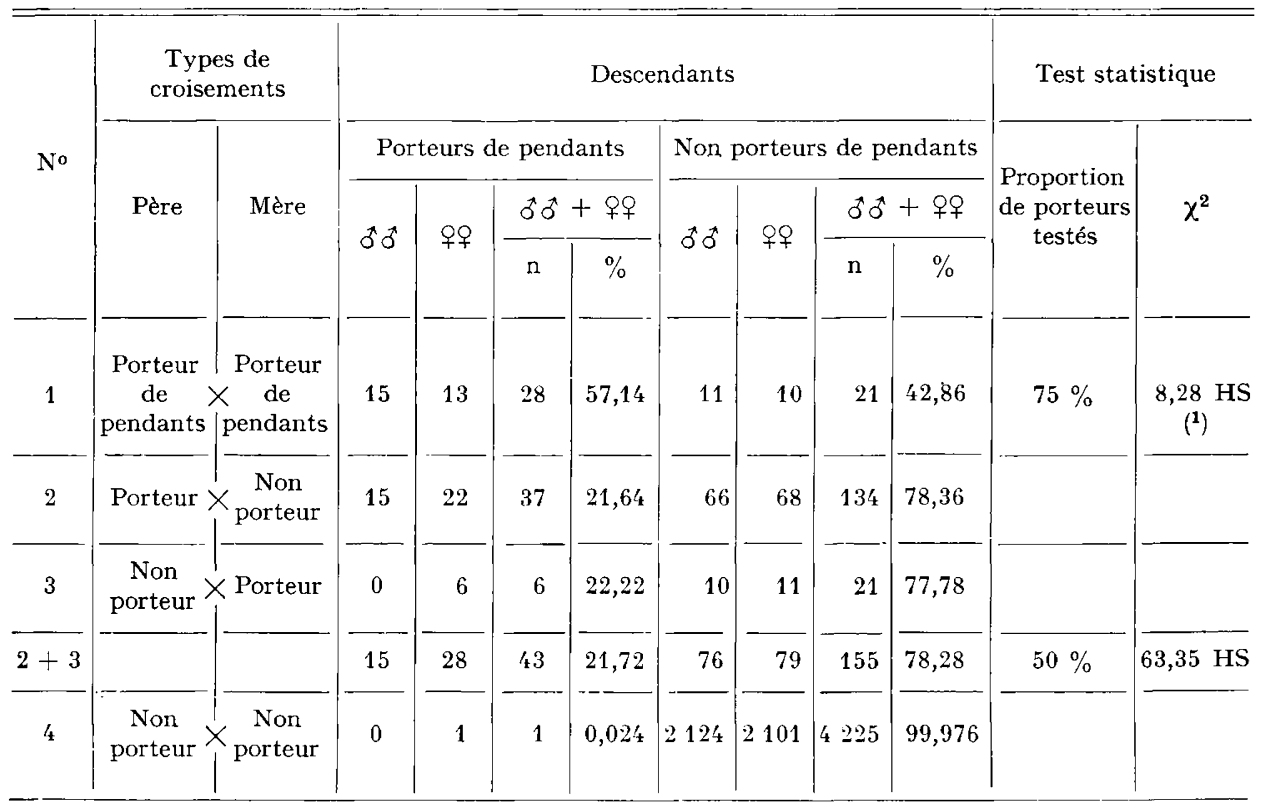

(1) HS : écart hautement significatif (aı seuil 0,01).

\section{3. - Type de déterminisme héréditaire}

Les anciens auteurs [VASIN, I928, repris par SERRA, I948] considéraient que ces formations, présentes dans de nombreuses races, étaient en réalité de deux types différents ayant chacun leur étiologie propre : les pendants et les peignes.

Bien que, malheureusement, on n'ait pas noté individuellement la forme des attributs présentée par les porteurs, - ce qui aurait permis de prouver l'unicité de l'étiologie en comparant l'expressivité chez les parents et les descendants on ne peut guère mettre celle-ci en doute. Ėn effet la fig. I montre en premier lieu la continuité de l'expressivité depuis le tout petit pendant jusqu'au peigne. En second lieu on pourra voir dans la fig. $2 b$ que chez un même individu les 2 types (peigne et pendant) peuvent être associés, ce qui, avec les faibles fréquences observées, serait impensable s'il s'agissait de caractères régis par deux facteurs indépendants.

\section{4. -- Les paramètres génétiques}

Si l'on peut rester dans un cadre monofactoriel mendélien on remarque que 1'estimation des 3 paramètres qui donnent la solution du problème (la pénétrance chez 1'homozygote $: w_{1}$, la pénétrance chez l'hétérozygote : $w_{2}$ et la fréquence génique $: q$ 

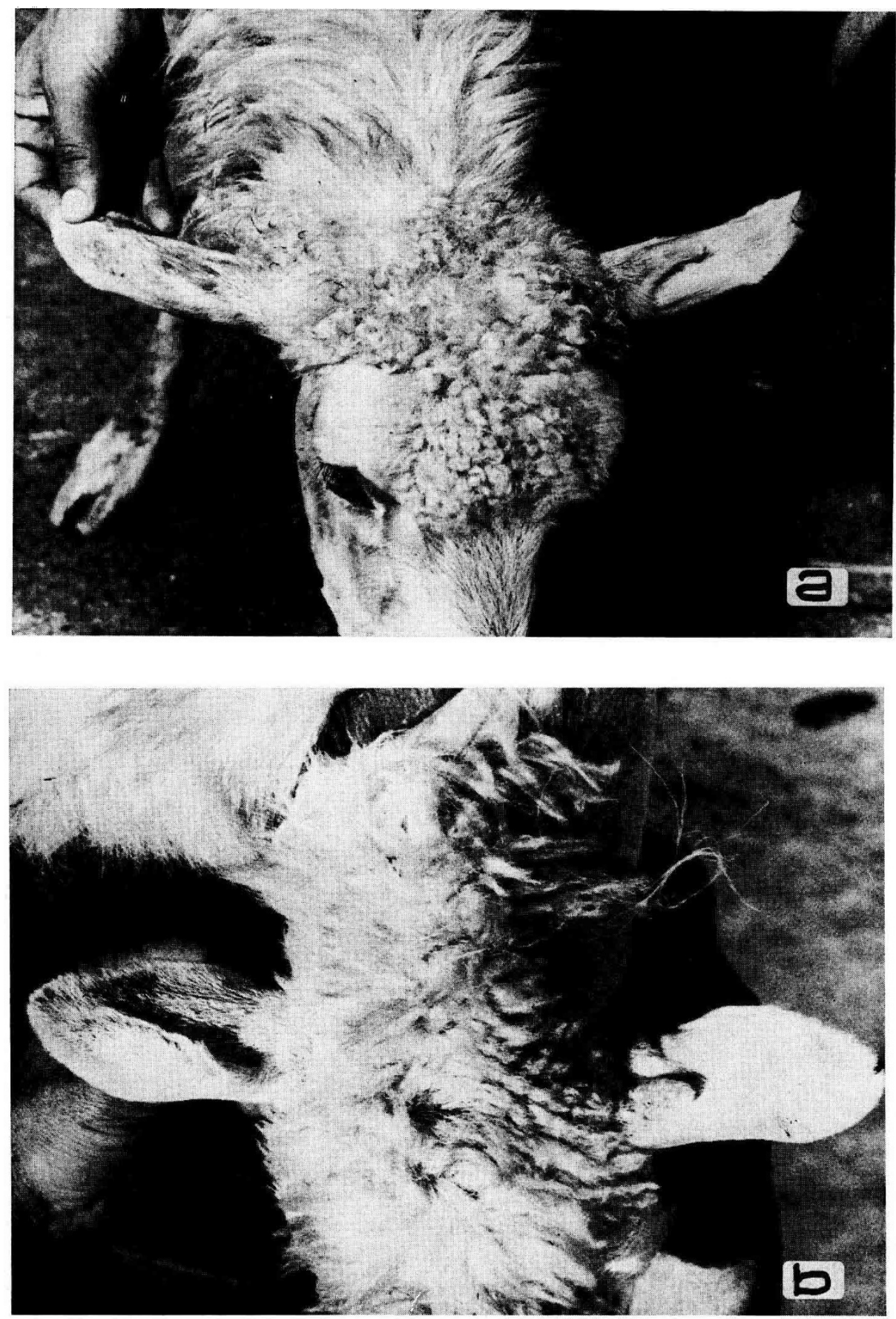

Fig. 2. - Types d'association des pendants chez le même animal

a) Un pendant très petit à droite ; un pendant long à gauche ;

b) Un " peigne " sur l'oreille droite; un pendant sur l'oreille gauche. 
dans la population) est possible à partir des données contenues dans le tableau $\mathrm{I}$. Il faut, pour cela, admettre que la fréquence $q$ est à l'équilibre, que les pénétrances sont les mêmes dans les 2 sexes et négliger les forces qui contribuent à maintenir l'équilibre, c'est-à-dire les coefficients de sélection différentiels.

Dans ces conditions, les 2 fréquences gamétiques du facteur en question $\delta$ chez les porteurs du caractère et $\delta^{\prime}$ chez les non porteurs s'écrivent :

et

$$
\delta=\frac{w_{1} q+w_{2}(\mathrm{I}-q)}{w_{1} q+2 w_{2}(\mathrm{I}-q)}
$$

$$
\delta^{\prime}=\frac{q^{2}\left(\mathrm{I}-w_{1}\right)+q(\mathrm{I}-q)\left(\mathrm{I}-w_{2}\right)}{q^{2}\left(\mathrm{I}-w_{1}\right)+2 q(\mathrm{I}-q)\left(\mathrm{I}-w_{2}\right)+(\mathrm{I}-q)^{2}}
$$

La probabilité $x$ d'apparition d'un porteur de pendants dans le croisement I du tableau I (porteur $\times$ porteur) est alors :

$$
x=\delta^{2} w_{1}+2 \delta(\mathbf{I}-\delta) w_{2}
$$

et $y$, la p尊babilité d'apparition d'un porteur de pendants dans les croisements 2 et 3 , du type (porteur $\times$ non porteur) s'écrit :

$$
y=\delta \delta^{\prime} w_{1}+w_{2}\left(\delta+\delta^{\prime}-2 \delta \delta^{\prime}\right)
$$

L'estimation par le maximum de vraisemblance donne:

et on a le système d'équation :

$$
x=\frac{2 \mathrm{I}}{49} \quad \text { et } \quad y=\frac{43}{\mathrm{I} 98}
$$

$$
\left\{\begin{array}{l}
\delta^{2} w_{1}+2 \delta(\mathrm{I}-\delta) w_{2}=\frac{2 \mathrm{I}}{49} \\
\delta \delta^{\prime} w_{1}+w_{2}\left(\delta+\delta^{1}-2 \delta \delta^{\prime}\right)=\frac{43}{\mathrm{I} 98}
\end{array}\right.
$$

Si l'on admet (comme la fréquence phénotypique très basse dans la population de l'île le suggère, la pénétrance étant de l'ordre de 0,5 au moins chez l'homozygote), que la fréquence du gène dans la population est très faible, on peut, en première approximation, poser $\delta=0,5$ et $\delta^{\prime}=0$ et le système devient :

dont la solution immédiate est :

$$
\left\{\begin{array}{l}
\frac{w_{1}}{4}+\frac{w_{2}}{2}=\frac{2 \mathrm{I}}{49} \\
\frac{w_{2}}{2}=\frac{43}{\mathrm{I} 9^{8}}
\end{array}\right.
$$

$$
\begin{aligned}
& w_{1}=0,8456 \\
& w_{2}=0,4342
\end{aligned}
$$

En fait, les hypothèses $\delta=0,5$ et $\delta^{\prime}=0$ signifient simplement que l'on suppose tous les porteurs hétérozygotes, les non-porteurs étant homozygotes normaux.

Pour, maintenant, estimer la fréquence $q$ du gène dans la population, on peut calculer tout d'abord $\delta^{\prime}$ à partir des résultats des croisements entre animaux non porteurs ( $\mathbf{n}^{0} 4$ du tableau I), où la probabilité $z$ d'apparition d'un porteur s'écrit :

$$
z=\delta^{\prime 2} w_{1}+2 \delta^{\prime}\left(\mathrm{I}-\delta^{\prime}\right) w_{2}
$$




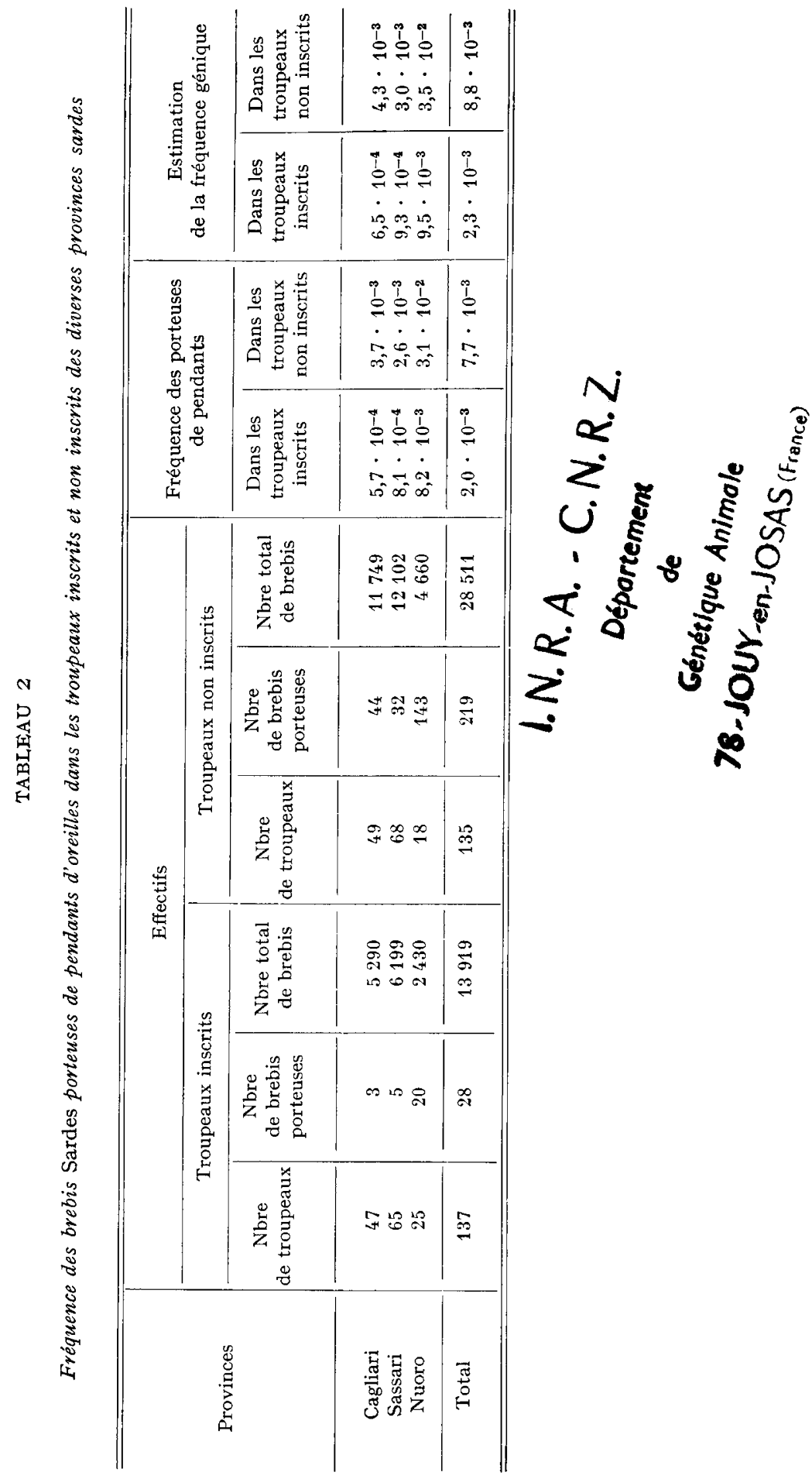


et qui, comme précédemment, a pour estimation par le maximum de vraisemblance la fréquence de porteurs observée :

$$
\frac{I}{4226}=0,00024
$$

En remplaçant $w_{1}, w_{2}$ et $q$ par leurs estimées, on a alors l'équation : $0,0226 \delta^{\prime 2}-0,8684 \delta^{\prime}+0,00024=0$ qui permet de calculer : $\delta^{\prime}=0,00028$

En reportant cette valeur dans (2), on obtient une équation de deuxième degré en $q$ qui s'écrit :

d'où l'on tire :

$$
0,4 \text { II } 40 q^{2}-0,56555 q+0,00028=0
$$

$$
q=0,000495 \not 5 \cdot \mathrm{IO}^{-4}
$$

\section{5. - La variation de la fréquence dans l'île}

Dans le tableau 2 on donne les fréquences de brebis porteuses de l'anomalie pour les diverses sous-populations Sardes des différentes provinces, dans les troupeaux inscrits et non inscrits.

On peut remarquer, $q$ étant toujours très faible, qu'on n'observera pratiquement que des hétérozygotes. On peut donc déduire une estimée de la fréquence génique en divisant la fréquence des porteuses par le double de la pénétrance chez les hétérozygotes. C'est ce qui a été fait dans les deux colonnes de droite du tableau 2.

\section{TABLEAU 3}

Comparaison des fréquences du gène de pendants d'oreilles entre troupeaux inscrits et non inscrits

\begin{tabular}{c|c|c|c}
\hline Inscrits & Non inscrits \\
\cline { 2 - 4 } & Cagliari + Sassari & Nuoro & Total non inscrits \\
\hline Cagliari + Sassari & HS & HS & HS \\
\hline Total inscrits & HS & HS \\
\hline
\end{tabular}

HS : écart hautement significatif.

Une simplification dans la comparaison des fréquences entre les diverses populations peut être introduite par un test préalable intra-troupeaux inscrits ou non inscrits entre provinces. On remarque que l'écart n'est pas significatif entre les provinces de Cagliari et Sassari. On peut donc ainsi regrouper les statistiques concernant ces deux provinces, dans les comparaisons qui sont données schématiquement dans les tableaux 3 et 4 . 
TABLEAU 4

Comparaison des fréquences du gène de pendants d'oreilles entre les provinces de Nuoro et de Sassari-Cagliari

\begin{tabular}{c|c|c}
\hline \multirow{2}{*}{ Sassari + Cagliari } & \multicolumn{2}{|c}{ Nuoro } \\
\cline { 2 - 3 } & Troupeaux inscrits & Troupeaux non inscrits \\
\hline Troupeaux inscrits & HS & \\
\hline Troupeaux non inscrits & & HS \\
\hline
\end{tabular}

HS : écart hautement significatif.

\section{DISCUSSION}

Les estimations des pénétrances sont inférieures à l'unité, elles reflètent bien la situation d'expressivité variable illustrée par la fig. I. Il est dommage que la notation du degré d'expressivité chez chaque porteur n'ait pu être faite, ce qui aurait permis d'apporter d'intéressantes précisions.

Quant aux comparaisons entre les fréquences géniques, on voit tout d'abord que l'estimation faite par comptage dans les provinces de Cagliari et Sassari' $\left(8,5 \cdot \mathrm{IO}^{-4}\right.$ correspond à peu près à celle que l'on a pu faire dans les troupeaux expérimentaux de $1^{\prime}$ Institut $\left(5 \cdot \mathrm{IO}^{-4}\right)$ dont les éléments provenaient précisément de troupeaux inscrits de ces deux provinces. Il est même normal que cette fréquence soit un peu inférieure car elle est principalement basée sur des croisements de non-porteurs par non-porteurs $\left({ }^{1}\right)$.

$\mathrm{Si}$, maintenant, l'on compare les sous-populations insulaires groupées selon leur province et leur relation avec le Flock-book (inscrites ou non inscrites) on note deux différences significatives : tout d'abord entre troupeaux inscrits et troupeaux non inscrits, la fréquence dans ces derniers étant fort supérieure ; en deuxième lieu entre la province de Nuoro et les deux autres provinces de l'île, aussi bien pour les troupeaux inscrits que non inscrits.

Ces différences peuvent s'expliquer par les migrations de béliers mises en lumière dans l'étude démographique déjà évoquée (LAUVERGNE et al. 1973). Les troupeaux inscrits de Sassari et de Cagliari subissent une influence du centre primaire de diffusion de Barumini bien plus forte que ceux de la province de Nuoro. Leur fréquence s'est alignée assez facilement sur celle de ce centre où il n'est pas de mode de conserver

(1) On constate en effet, en particulier lors des expériences de sélection sur la pénétrance d'un gène' que la valeur de cette pénétrance diffère selon que l'on utilise des porteurs de même génotype sains ou tarés. Les estimations auxquelles nous nous sommes livrés plus haut sont donc en réalité des moyennes plus ou moins bien pondérées de ces pénétrances. Elles seraient sans doute différentes si le rapport entre les effectifs des différents croisements analysés variaient. Avec le degré d'approximation qui est le nôtre il a paru inutile d'en tenir compte ici. 
des béliers avec pendants. Quant aux troupeaux non inscrits, ils ne subissent l'influence du centre de Barumini que d'une manière atténuée, surtout si ils sont isolés géographiquement, comme c'est le cas dans la province de Nuoro.

Cette situation se retrouve pour la fréquence des pendeloques dont les estimations varient peu entre les troupeaux privés regroupés par régions dans les 2 provinces de Cagliari et de Sassari $(0,84 \mathrm{I} ; 0,870 ; 0,876 ; 0,80 \mathrm{or} ; 0,945 ; 0,897 ; 0,872$ pour la fréquence de l'allèle normal récessif du gène donnant les pendeloques, CASU et al, I97I). Malheureusement cet article ne donne pas d'estimées pour la province de Nuoro, elles eussent été fort instructives.

Remarquons, pour terminer, que les faibles fréquences phénotypiques et géniques n'autorisent guère une étude plus détaillée troupeau par troupeau et ne permettent pas de faire des comparaisons entre animaux de différents génotypes pour les caractères pouvant influencer le coefficient de sélection ou avoir un intérêt zootechnique, comme cela avait pu être fait à propos des pendeloques (cf. l'article cité plus haut).

\section{CONCLUSION}

La manière d'aborder l'étude du comportement du gène de pendants d'oreille dans la race Sarde est évidemment sommaire. Il est vrai que les données dont nous disposions étaient peu nombreuses. On a vu en outre quelles ne permettaient pas l'étude de l'expressivité.

Quant à la fréquence génique, ses variations semblent explicables en gros par le système d'échanges de béliers que nous avons mis en évidence dans une précédente étude. Malheureusement la faible fréquence du facteur considéré interdit l'analyse détaillée du phénomène migratoire.

Reçu pour publication en octobre 1973.

\section{REMERCIEMENTS}

Les remarques de MM. B. Vissac et P. Mérat ont beaucoup aidé à la mise sur pied du texte que nous présentons.

\section{SUMMARY}

\section{EAR PENDANTS IN SARDINIAN SHEEP}

In Sardinian sheep, ear pendants ("orechiete 》) have different shapes, positions and associations. Their presence may be explained by the action of a single autosomal locus with variable expressivity and incomplete penetrance. The penetrance is the same in both sexes and equals 0.84 in the homozygotes and 0.43 in the heterozygote.

From a sample deriving from crosses between animals themselves not having pendants, from the herds of the Institut Zootechnique et Fromager Sarde the gene frequency has been found to be very low $\left(q=5 \times \mathrm{ro}^{-4}\right)$. By chance this estimate is close to that obtained from herds in the two provinces of Cagliari and Sassari $\left(q=8,5 \times 1 \mathrm{ro}^{-4}\right)$, the source of the animals in the Institute herd. In the other Sardinian province of Nuoro, on the other hand, the frequency is signi- 
ficantly different from that observed in Cagliari and Sassari. This might arise from the fact that, as an earlier study has shown (LAUVERGNe et al., 1973,), the herds in these two provinces have been more strongly influenced genetically by the centre of Barumini. In Nuoro moreover, the number of rams born outside the province is much less. In all three provinces, the gene frequencies in those herds not inscribed in the herd book are significantly higher than in those which are so inscribed. The difference might be explained by the much wider choice of rams available in the latter.

\section{RÉFÉRENCES BIBLIOGRAPHIQUES}

Casu S., Boyazoglu J. G., Lauvergne J. J., I97o. Hérédité des pendeloques dans la race ovine Sarde. Ann. Génét. Sél. anim., 2, 249-26r.

Casu S., Boyazoglu J. G., Lauvergne J. J., i97i. "Pendants " d'oreilles dans la race ovine Sarde. Congrès Européen de Zootechnie, Thème II, 3 p.

Lauvergne J.J., Boyazoglu J. G., Carta R. et Casu S., 1973. Caractéristiques démographiques de la race ovine Sarde. Ann. Génét. Sél. anim., 5, 53-72.

Serra J. A., r948. Génétique du mouton. Publ. Junta Pecuar, Lisboa, Ser. A, n⿳0 I.

VAsin B., I928. Genetika ovets. I. Nasledovamie obraski i pejni, Moskva, (cité par SerRA, r948). 